REPORTS ON MATHEMATICAL LOGIC

52 (2017), 45-56

doi:10.4467/20842589RM.17.002.7140

\title{
Ahmad KARIMI
}

\section{A NON-SELF-REFERENTIAL PARADOX IN EPISTEMIC GAME THEORY}

\begin{abstract}
A b s t r a c t. The beliefs of other people about our own beliefs affect our decision making strategies (and beliefs). In epistemic game theory, player's beliefs about other players' beliefs are formalized, and the way how people may reason about other players (before they make their final choice in a game) is explored. In this paper, we introduce a non-self-referential paradox (called "Yablo-like Brandenburger-Keisler paradox") in epistemic game theory which shows that modeling players' epistemic beliefs and assumptions in a complete way is impossible. We also present an interactive temporal (belief and) assumption logic to give an appropriate formalization for this Yablo-like Brandenburger-Keisler paradox. Formalizing the new paradox turns it into a theorem in the interactive temporal assumption logic.
\end{abstract}

Received 27 April 2016

Keywords and phrases: non-self-referential paradox, Brandenburger-Keisler paradox, Yablo's paradox, temporal assumption logic, interactive models. AMS subject classification: 03B42, 03B44, $03 A 05$. 


\section{Introduction}

Adam Brandenburger and H. Jerome Keisler introduce a two-person and self-referential paradox in epistemic game theory [5]. They show that the following configuration of beliefs can not be represented: Ann believes that Bob assumes that Ann believes that Bob's assumption is wrong. The Brandenburger-Keisler paradox (BK paradox) shows impossibility of completely modeling players' epistemic beliefs and assumptions. Brandenburger and Keisler present a modal logic interpretation of the paradox [5]. They introduce two modal operators intended to represent the agents' beliefs and assumptions. In [17] Pacuit approaches the BK paradox from a modal logical perspective and presents a detailed investigation of the paradox in neighborhood models and hybrid systems. In particular, he shows that the paradox can be seen as a theorem of an appropriate hybrid logic.

The BK paradox is essentially a self-referential paradox and similarly to any other paradox of the same kind can be analyzed from a category theoretical or algebraic point of view. In [1] Abramsky and Svesper analyze the BK paradox in categorical context. They present the paradox as a fixed-point theorem, which can be carried out in any regular category, and show how it can be reduced to a relational form of the one-person diagonal argument due to Lawvere [15] where he gave a simple form of the (oneperson) diagonal argument as a fixed-point lemma in a very general setting.

Başkent in [2] approaches the BK paradox from two different perspectives: non-well-founded set theory and paraconsistent logic. He shows that the paradox persists in both frameworks for category-theoretical reasons, but with different properties. Başkent makes the connection between selfreferentiality and paraconsistency.

On the other hand, Stephen Yablo [21] introduce a logical paradox in 1993 that is similar to the liar paradox where he used an infinite sequence of statements. Every statement in the sequence refers to the truth values of the later statements. Therefore, it seems this paradox avoids self-reference.

In this paper, we present a non-self-referential multi-agent paradox in epistemic game theory which we call "Yablo-like Brandenburger-Keisler paradox". In an interactive temporal assumption logic we study interactive reasoning: how agents reason about the beliefs of other agents over time. We also present an interactive temporal (belief and) assumption logic to give an appropriate formalization for this Yablo-like Brandenburger-Keisler para- 
dox. Formalizing the new paradox in the interactive temporal assumption logic turns it into a theorem in this logic.

\section{Brandenburger-Keisler Paradox}

Brandenburger and Keisler introduced the following two person Russellstyle paradox $[2,5,17]$. Beliefs and assumptions are two main concepts involved in the statement of the paradox. An assumption is the strongest belief. Suppose there are two players, Ann and Bob, and consider the following description of beliefs:

Ann believes that Bob assumes that Ann believes that Bob's assumption is wrong.

A paradox arises when one asks the question "Does Ann believe that Bob's assumption is wrong?" Suppose that answer to the above question is "yes". Then according to Ann, Bob's assumption is wrong. But, according to Ann, Bob's assumption is Ann believes that Bob's assumption is wrong. However, since the answer to the above question is "yes", Ann believes that this assumption is correct. So Ann does not believe that Bob's assumption is wrong. Therefore, the answer to the above question must be "no". Thus, it is not the case that Ann believes that Bob's assumption is wrong. Hence Ann believes Bob's assumption is correct. That is, it is correct that Ann believes that Bob's assumption is wrong. So, the answer must have been "yes". This is a contradiction!

Brandenburger and Keisler [5] introduce belief models for two players to present their impossibility results. In a belief model, each player has a set of states, and each state for one player has beliefs about the states of the other player. We assume the reader is familiar with the belief models and formulation of BK paradox in modal logic, but for the sake of accessibility, we list the main notations, definitions and theorems which will be referred to later on. For more details, we refer the reader to $[5,17]$.

Definition 1. A belief model is a two-sorted structure

$$
\mathcal{M}=\left(U^{a}, U^{b}, P^{a}, P^{b}, \ldots\right)
$$


where $U^{a}$ and $U^{b}$ are the nonempty universe sets (for the two sorts), $P^{a}$ is a proper subset of $U^{a} \times U^{b}, P^{b}$ is a proper subset of $U^{b} \times U^{a}$, and $P^{a}, P^{b}$ are serial, that is, the sentences

$$
\forall x \exists y P^{a}(x, y) \text { and } \forall y \exists x P^{b}(y, x)
$$

hold. To simplify notation, $x$ is always a variable of sort $U^{a}$ and $y$ is a variable of sort $U^{b}$. The members of $U^{a}$ and $U^{b}$ are called states for Ann and Bob, respectively. $P^{a}$ and $P^{b}$ are called possibility relations. We say $x$ believes a set $Y \subseteq U^{b}$ if $\left\{y: P^{a}(x, y)\right\} \subseteq Y$, and $x$ assumes $Y$ if $\left\{y: P^{a}(x, y)\right\}=Y$.

For an arbitrary structure $\mathcal{N}=\left(U^{a}, U^{b}, P^{a}, P^{b}, \ldots\right)$, by the first order language for $\mathcal{N}$ we mean the two-sorted first order logic with sorts for $U^{a}$ and $U^{b}$ and symbols for the relations in the vocabulary of $\mathcal{N}$. Given a first order formula $\varphi(u)$ whose only free variable is $u$, the set defined by $\varphi$ in $\mathcal{N}$ is the set $\{u: \varphi(u)$ is true in $\mathcal{N}\}$. In general, by a language for $\mathcal{N}$ we will mean a subset of the set of all formulas of the first order language for $\mathcal{N}$. Given a language $\mathcal{L}$ for $\mathcal{N}$, we let $\mathcal{L}_{a}, \mathcal{L}_{b}$ be the families of all subsets of $U^{a}, U^{b}$ respectively which are defined by formulas in $\mathcal{L}$. Also, The diagonal formula $D(x)$ is the first order formula

$$
\forall y\left(P^{a}(x, y) \rightarrow \neg P^{b}(y, x)\right) .
$$

This is the formal counterpart to the intuitive statement: "Ann believes that Bob's assumption is wrong."

Definition 2. Let $\mathcal{M}$ be a belief model, and let $\mathcal{L}$ be a language for it. The model $\mathcal{M}$ is complete for $\mathcal{L}$ if each nonempty set $Y \in \mathcal{L}_{b}$ is assumed by some $x \in U^{a}$, and each nonempty $X \in \mathcal{L}_{a}$ is assumed by some $y \in U^{b}$.

In their paper [5], Brandenburger and Keisler prove that there is no belief model which is complete for a language $\mathcal{L}$ which contains the tautologically true formulas, $D(x), \forall y P^{b}(y, x)$ and formula built from the formal counterpart of the BK paradox. They also present modal formulations of the BK paradox by presenting a specific modal logic, called interactive assumption logic, with two modal operators for "belief" and "assumption". For semantical interpretations Brandenburger and Keisler use Kripke models [5]. Pacuit [17] presents a detailed investigation of the BK paradox in neighborhood models and in hybrid systems. He shows that the paradox can be seen as a theorem of an appropriate hybrid logic. 


\section{Yablo's Paradox}

To counter a general belief that all the paradoxes stem from a kind of circularity (or involve some self-reference, or use a diagonal argument) Stephen Yablo designed a paradox in 1993 that seemingly avoided self-reference $[20,21]$. Since then much debate has been sparked in the philosophical community as to whether Yablo's Paradox is really circular-free or involves some circularity (at least hidden or implicitly); see e.g. [3, 6-8, 12, 13, 18, 19, 22]. Unlike the liar paradox, which uses a single sentence, this paradox applies an infinite sequence of statements. There is no consistent way to assign truth values to all the statements, although no statement directly refers to itself. Yablo considers the following sequence of sentences $\left\{S_{i}\right\}$ :

$$
\begin{aligned}
& S_{1}: \forall k>1 ; S_{k} \text { is untrue, } \\
& S_{2}: \forall k>2 ; S_{k} \text { is untrue, } \\
& S_{3}: \forall k>3 ; S_{k} \text { is untrue, }
\end{aligned}
$$

The paradox follows from the following deductions. Suppose $S_{1}$ is true. Then for any $k>1, S_{k}$ is not true. Specially, $S_{2}$ is not true. Also, $S_{k}$ is not true for any $k>2$. But this is exactly what $S_{2}$ says, hence $S_{2}$ is true after all. Contradiction! Suppose then that $S_{1}$ is false. This means that there is a $k>1$ such that $S_{k}$ is true. But we can repeat the reasoning, this time with respect to $S_{k}$ and reach a contradiction again. No matter whether we assume $S_{1}$ to be true or false, we reach a contradiction. Hence the paradox. Yablo's paradox can be viewed as a non-self-referential liar's paradox; it has been used to give alternative proof for Gödel's first incompleteness theorem $[9,16]$. Recently in $[10,11]$, formalization of Yablo's paradox and its different versions in Linear Temporal Logic (LTL) yield genuine theorems in this logic. 


\section{A Non-Self-Referential Paradox in Epistemic Game Theory}

In this section, we present a non-self-referential paradox in epistemic game theory. Unlike the BK paradox which uses one single statement on belief and assumption of agents, this paradox consists of two sequences of players (or agents) and a sequence of statements of agent's belief and assumptions. Indeed, this paradox shows that not every configuration of agents' beliefs and assumptions can be represented. Let us consider two infinite sequence of players $\left\{A_{i}\right\}$ and $\left\{B_{i}\right\}$, with the following description of beliefs:

$\begin{array}{cc}A_{1} & B_{1} \\ A_{2} & B_{2} \\ A_{3} & B_{3} \\ \vdots & \vdots\end{array}$

For all $i, A_{i}$ believes that $B_{i}$ assumes that for all $j>i, A_{j}$ believes that $B_{j}$ 's assumption is wrong.

A paradox arises when one asks the question "Does $A_{1}$ believe that $B_{1}$ 's assumption is wrong?"

Suppose that the answer to the above question is "no". Thus, it is not the case that $A_{1}$ believes that $B_{1}$ 's assumption is wrong. Hence $A_{1}$ believes $B_{1}$ 's assumption is correct. That is, it is correct that for all $j>1, A_{j}$ believes that $B_{j}$ 's assumption is wrong. Specially, $A_{2}$ believes that $B_{2}$ 's assumption is wrong. On the other hand, since for all $j>2, A_{j}$ believes that $B_{j}$ 's assumption is wrong, one can conclude that $A_{2}$ believes $B_{2}$ 's assumption is correct. Therefore, at the same time $A_{2}$ believes that $B_{2}$ 's assumption is both correct and wrong. This is a contradiction!

If the answer to the above question is "yes". Then according to $A_{1}$, $B_{1}$ 's assumption is wrong. But, according to $A_{1}, B_{1}$ 's assumption is that "for all $j>1, A_{j}$ believes that $B_{j}$ 's assumption is wrong". Thus, there is $k>1$ for which $A_{k}$ believes that $B_{k}$ 's assumption is correct. Now we can apply the same reasoning we used before about $A_{k}$ and $B_{k}$ to reach the contradiction! Hence the paradox. This paradox is a non-self-referential multi-agent version of the BK paradox. 


\section{Interactive Temporal Assumption Logic}

The beliefs of other people about our own beliefs affect our decision making strategies (and beliefs). These beliefs may change over the time. Belief revision is the process of changing beliefs to take into account a new piece of information and is a topic of much interest in theoretical computer science and logic. Assumption Logic is a multi-modal logic which is suitable for formalization of beliefs (and assumptions) of agents in a multi-agent system. This logic was first introduced by Bonanno for belief revision [4] and was simplified by Brandenburger and Keisler to formalize their paradox in epistemic game theory [5]. In order to reformulate their two-player impossibility result in modal logic setting, Brandenburger and Keisler present interactive version of this assumption logic with two operators $\square^{a b}$ and $\varnothing^{a b}$ standing for belief and assumption Ann about Bob, respectively. As an extension, temporal assumption logic is a formalism in which one can study changing the agents' beliefs over time. In this section, we aim to introduce an interactive Temporal Assumption Logic (iTAL) to present an appropriate formulation of the non-self-referential Yablo-like BK paradox.

The interactive temporal assumption language $\mathcal{L}_{i T A L}$ contains individual propositional symbols, the propositional connectives, the linear-time operators $\bigcirc, \square, \diamond$ and the epistemic operators "Believe" and "Assumption": for each pair of players $i j$ among Ann and Bob, the operator $B^{i j}$ will be beliefs for player $i$ about $j$, and $A^{i j}$ is the assumption for $i$ about $j$. In words, $B^{i j} \phi$ means that the agent $i$ believes $\phi$ about $j$, and $A^{i j} \phi$ is that the agent $i$ assumes $\phi$ about agent $j$. The temporal operators $\bigcirc, \square$, and $\diamond$ are called next time, always (or henceforth), and sometime (or eventuality) operators, respectively. Formulas $\bigcirc \varphi, \square \varphi$, and $\diamond \varphi$ are typically read "next $\varphi$ ", "always $\varphi$ ", and "sometime $\varphi$ ". We note that $\diamond \varphi \equiv \neg \square \neg \varphi$. For more details on linear temporal logic we refer the reader to [14].

Definition 3. Formulas in $\mathcal{L}_{i T A L}$ are defined as follows:

$$
\phi:=p|\neg \phi| \phi \wedge \psi|\bigcirc \phi| \square \phi\left|B^{i j} \phi\right| A^{i j} \phi .
$$

For semantical interpretations we introduce an appropriate class of Kripke models.

Definition 4. An iTAL-Model is a Kripke structure

$$
\mathcal{W}=\left(W, \mathbb{N},\left\{P_{n}: n \in \mathbb{N}\right\}, U^{a}, U^{b}, V\right),
$$


where $W$ is nonempty set, $\mathbb{N}$ is the set of natural numbers, for each $n \in$ $\mathbb{N}, P_{n}$ is a binary relation $P_{n} \subseteq W \times W$ and $U^{a}, U^{b}$ are disjoint sets such that $\left(U^{a}, U^{b}, P_{n}^{a}, P_{n}^{b}\right)$ is a belief model, where $U^{a} \cup U^{b}=W, P_{n}^{a}=P_{n} \cap U^{a} \times U^{b}$, and $P_{n}^{b}=P_{n} \cap U^{b} \times U^{a}$. $V:$ Prop $\rightarrow 2^{\mathbb{N} \times W}$ is a function mapping to each propositional letter $p$ the subset $V(p)$ of Cartesian product $\mathbb{N} \times W$. Indeed, $V(p)$ is the set of pairs $(n, w)$ such that $p$ is true in the world $w$ at the moment $n$.

The satisfiability of a formula $\varphi \in \mathcal{L}_{i T A L}$ in a model $\mathcal{W}$, at a moment of time $n \in \mathbb{N}$ in a world $w \in W$, denoted by $\mathcal{W}_{n}^{w} \Vdash \varphi$ (in short; $(n, w) \Vdash \varphi$ ), is defined inductively as follows:

- $(n, w) \Vdash p \Longleftrightarrow(n, w) \in V(p)$ for $p \in$ Prop,

- $(n, w) \Vdash \neg \varphi \Longleftrightarrow(n, w) \Vdash \varphi$,

- $(n, w) \Vdash \varphi \wedge \psi \Longleftrightarrow(n, w) \Vdash \varphi$ and $(n, w) \Vdash \psi$,

- $(n, w) \Vdash \bigcirc \varphi \Longleftrightarrow(n+1, w) \Vdash \varphi$,

- $(n, w) \Vdash \square \varphi \Longleftrightarrow \forall m \geq n(m, w) \Vdash \varphi$,

- $\left.(n, w) \Vdash B^{i j} \varphi \Leftrightarrow(n, w) \Vdash \mathbf{U}^{i} \wedge \forall z\left[\left(P_{n}^{i}(w, z) \wedge(n, z) \Vdash \mathbf{U}^{j}\right) \rightarrow(n, z) \Vdash \varphi\right]\right)$,

- $\left.(n, w) \Vdash A^{i j} \varphi \Leftrightarrow(n, w) \Vdash \mathbf{U}^{i} \wedge \forall z\left[\left(P_{n}^{i}(w, z) \wedge(n, z) \Vdash \mathbf{U}^{j}\right) \leftrightarrow(n, z) \Vdash \varphi\right]\right)$.

Let $x$ has sort $U^{a}, y$ has sort $U^{b}$ and $\varphi$ is a statement about $y$. Intuitively, $(n, x) \Vdash B^{a b} \varphi$ says that "in time $n, x$ believes $\varphi(y)$ ", and $(n, x) \Vdash A^{a b} \varphi$ says that "in time $n, x$ assumes $\varphi(y)$ ". A formula is valid for $V$ in $\mathcal{W}$ if it is true at all $w \in W$, and satisfiable for $V$ in $\mathcal{W}$ if it is true at some $w \in W$. In an interactive assumption model $\mathcal{W}$, we will always suppose that $\mathbf{D}$ is a propositional symbol, and $V$ is an evaluation in $\mathcal{W}$ such that $V(\mathbf{D})$ is the set

$$
D=\left\{(n, x) \in \mathbb{N} \times W:(\forall y \in W)\left[P_{n}^{a}(x, y) \rightarrow \neg P_{n}^{b}(y, x)\right]\right\} .
$$

The property which defines set $D$ (stands for Diagonal) is our formal counterpart to the intuitive statement "Ann believes Bob's assumption is wrong". Note that, as pointed out by Brandenburger and Keisler [5] and by Pacuit [17], the intuitive statement contains the word "believes", but the formula is not of the form $x$ believes $\varphi(y)$. 
In the rest of paper, we present our formulation of the non-self-referential Yablo-like BK paradox in the interactive temporal assumption setting. The thought is that we can make progress by thinking of the sequences of agents in Yablo-like BK paradox not as infinite families of agents but as a two individual agents that their belief and assumption can be evaluated in lots of times (temporal states) in an epistemic temporal model. Thus, the emergence of the Yablo-like BK paradox should be the same as the derivability of a particular formula in the epistemic temporal logic.

Let us have a closer look at the Yablo-like BK paradox:

For all $i, A_{i}$ believes that $B_{i}$ assumes that for all $j>i, A_{j}$ believes that $B_{j}$ 's assumption is wrong.

Now suppose that there are two players, namely Ann and Bob. Assume that $A_{i}$ and $B_{i}$ are the counterparts of Ann and Bob in the $i^{\text {th }}$ temporal state. Then infinitely many statements in the Yablo-like BK paradox can be represented in just one single formula using temporal tools:

\section{$\square[$ Ann believes that Bob assumes that}

$(\bigcirc \square($ Ann believes that Bob's assumption is wrong $))]$

The interpretation of above formula in English can be seen as: "Always it is the case that Ann believes that Bob assumes from the next time henceforth that Ann believes that Bob's assumption is wrong". We note that Ann and Bob in each state refer to their belief and assumptions in the next temporal states; not the state that they are in.

Theorem 5. In an interactive temporal assumption model $\mathcal{W}$, if $A^{a b} \mathbf{U}^{b}$ is valid, then

$$
\square\left[B^{a b} A^{b a}(\bigcirc \square \mathbf{D})\right] \longrightarrow \square \mathbf{D}
$$

will be a valid formula in $\mathcal{W}$.

Proof. Assume $A^{a b} \mathbf{U}^{b}$ is valid, for all $l \in \mathbb{N}$ and $x \in W,(l, x) \Vdash A^{a b} \mathbf{U}^{b}$. This means, for all $l \in \mathbb{N}$ and $x \in W$,

$$
(l, x) \Vdash \mathbf{U}^{a} \wedge \forall y\left[P_{l}^{a}(x, y) \wedge(l, y) \Vdash \mathbf{U}^{b} \leftrightarrow(l, y) \Vdash \mathbf{U}^{b}\right] .
$$


Thus

$$
\forall l \forall x \quad(l, x) \Vdash \mathbf{U}^{a} \wedge \forall y\left[P_{l}^{a}(x, y)\right] .
$$

Now, for arbitrary $k \in \mathbb{N}$ and $\tilde{x} \in W$, let $(k, \tilde{x}) \Vdash \square\left[B^{a b} A^{b a}(\bigcirc \square \mathbf{D})\right]$; we show that $(k, \tilde{x}) \Vdash \square \mathbf{D}$. To this end, for a moment, suppose that $(k, \tilde{x}) \Vdash$ $\square \mathbf{D}$. So, there is $m \geq k$ for which $(m, \tilde{x}) \forall \mathbf{D}$. We can assume that $m=k$. Thus, $(k, \tilde{x}) \Vdash \neg \mathbf{D}$ which means that $\exists \tilde{y}\left[P_{k}^{a}(\tilde{x}, \tilde{y}) \wedge P_{k}^{b}(\tilde{y}, \tilde{x})\right]$. By (1) for $l=k,(k, \tilde{x}) \Vdash \mathbf{U}^{a} \wedge \forall y\left[P_{k}^{a}(\tilde{x}, y)\right]$. Since $(k, \tilde{x}) \Vdash \square\left[B^{a b} A^{b a}(\bigcirc \square \mathbf{D})\right]$, then

$$
\begin{aligned}
\forall l & \geq k(l, \tilde{x}) \Vdash B^{a b} A^{b a}(\bigcirc \square \mathbf{D}) \\
\Longrightarrow \forall l & \geq k(l, \tilde{x}) \Vdash \mathbf{U}^{a} \wedge \forall y\left[P_{l}^{a}(\tilde{x}, y) \wedge(l, y) \Vdash \mathbf{U}^{b} \rightarrow(l, y) \Vdash A^{b a}(\bigcirc \square \mathbf{D})\right] \\
\Longrightarrow \forall l & \geq k \forall y(l, y) \Vdash A^{b a}(\bigcirc \square \mathbf{D}) \\
\Longrightarrow \forall l & \geq k \forall y\left((l, y) \Vdash \mathbf{U}^{b} \wedge \forall w\left[P_{l}^{b}(y, w) \wedge(l, w) \Vdash \mathbf{U}^{a} \leftrightarrow(l, w) \Vdash \bigcirc \square \mathbf{D}\right]\right) .
\end{aligned}
$$

Therefore, since $P_{k}^{b}(\tilde{y}, \tilde{x})$, one can conclude that $(k, \tilde{x}) \Vdash \bigcirc \square$ D. So, we have $\forall l \geq k+1(l, \tilde{x}) \Vdash \mathbf{D}$, thus $\forall l \geq k+1\left[P_{l}^{a}(\tilde{x}, \tilde{y}) \rightarrow \neg P_{l}^{b}(\tilde{y}, \tilde{x})\right]$. In particular, for $k+1, P_{k+1}^{a}(\tilde{x}, \tilde{y}) \rightarrow \neg P_{k+1}^{b}(\tilde{y}, \tilde{x})$. By $(1)$, since $P_{k+1}^{a}(\tilde{x}, \tilde{y})$ then $\neg P_{k+1}^{b}(\tilde{y}, \tilde{x})$. On the other hand, since $(k+1, \tilde{x}) \Vdash \bigcirc \square \mathbf{D}$, then $P_{k+1}^{b}(\tilde{y}, \tilde{x})$ which is a contradiction! Therefore, $(k, \tilde{x}) \Vdash \square \mathbf{D}$ which shows that

$$
\square\left[B^{a b} A^{b a}(\bigcirc \square \mathbf{D})\right] \longrightarrow \square \mathbf{D}
$$

is valid in $\mathcal{W}$.

Theorem 6. In any interactive temporal assumption model $\mathcal{W}$, if $A^{a b} \mathbf{U}^{b}$ and $B^{a b} A^{b a}\left(\mathbf{U}^{a}\right)$ are valid, then the formula

$$
\neg \square\left[B^{a b} A^{b a}(\bigcirc \square \mathbf{D})\right]
$$

will be valid.

Proof. If not, then $(k, \tilde{x}) \Vdash \square\left[B^{a b} A^{b a}(\bigcirc \square \mathbf{D})\right]$ for some sate $(k, \tilde{x})$. By Theorem $5,(k, \tilde{x}) \Vdash \square \mathbf{D}$ so $\forall l \geq k,(l, \tilde{x}) \Vdash \mathbf{D}$, in particular for $l=k$. Thus, $\forall y\left[P_{k}^{a}(\tilde{x}, y) \rightarrow \neg P_{k}^{b}(y, \tilde{x})\right]$. On the other hand, $(k, \tilde{x}) \Vdash B^{a b} A^{b a} \mathbf{U}^{\mathbf{a}}$. By the definitions of $B^{a b}$ and $A^{b a}$ on $\mathbf{U}^{\mathbf{a}}$, there is some $\tilde{y}$ such that the relation $\left[P_{k}^{a}(\tilde{x}, \tilde{y}) \wedge P_{k}^{b}(\tilde{y}, \tilde{x})\right]$ holds. So, $(k, \tilde{x}) \Vdash \neg \mathbf{D}$ which is a contradiction! Therefore, $\neg \square\left[B^{a b} A^{b a}(\bigcirc \square \mathbf{D})\right]$ is valid in $\mathcal{W}$. 


\section{Conclusions}

In game theory, the notion of a player's beliefs about the game player's beliefs about other players' beliefs arises naturally. We presented a nonself-referential paradox in epistemic game theory which we called "Yablolike Brandenburger-Keisler paradox". Arising the paradox shows that completely modeling the players' epistemic beliefs and assumptions is impossible. We formalized Yablo-like Brandenburger-Keisler paradox in the interactive temporal assumption logic and transformed it into a theorem in this logic.

\section{Acknowledgments}

I would like to thank the anonymous reviewers for helpful comments and suggestions.

\section{References}

[1] S. Abramsky and J. Zvesper, From Lawvere to Brandenburger-Keisler: interactive forms of diagonalization and self-reference, Journal of Computer and System Sciences 81 (2015), 799-812.

[2] C. Başkent, Some Non-classical Approaches to the Brandenburger-Keisler Paradox, Logic Journal of IGPL 23 (2015), 533-552.

[3] J.C. Beall, Is Yablo's Paradox Non-Circular? Analysis 61 (2001), 176-187.

[4] G. Bonanno, A Simple Modal Logic for Belief Revision, Synthese 147 (2005), 193-228.

[5] A. Brandenburger and H. Jerome Keisler, An Impossibility Theorem on Beliefs in Games, Studia Logica 84 (2006), 211-240.

[6] S. Bringsjord and B. van Heuveln, The 'Mental Eye' Defence of an Infinitized Version of Yablo's Paradox, Analysis 63 (2003), 61-70.

[7] O. Bueno and M. Colyvan, Yablo's Paradox and Referring to Infinite Objects, Australasian Journal of Philosophy 81 (2003), 402-412.

[8] O. Bueno and M. Colyvan, Paradox without Satisfaction, Analysis 63 (2003), $152-156$.

[9] C. Cieśliński and R. Urbaniak, Gödelizing the Yablo Sequence, Journal of Philosophical Logic 42 (2013), 679-695. 
[10] A. Karimi and S. Salehi, Diagonalizing by Fixed-Points, Preprint arXiv:1303.0730, 2014.

[11] A. Karimi and S. Salehi, Theoremizing Yablo's Paradox, Preprint arXiv:1406.0134, 2014.

[12] J. Ketland, Bueno and Colyvan on Yablo's paradox, Analysis 64 (2004), 165-172.

[13] J. Ketland, Yablo's Paradox and w-Inconsistency, Synthese 145 (2005), 295-302.

[14] F. Kröger and S. Merz, Temporal Logic and State Systems, Springer 2008, ISBN 9783540674016.

[15] F.W. Lawvere, Diagonal Arguments and Cartesian Closed Categories, Lecture Notes in Mathematics 92 (1969), 134-145.

[16] G. Leach-Krouse, Yablifying the Rosser Sentence, Journal of Philosophical Logic 43 (2014), 827-834.

[17] E. Pacuit, Understanding the Brandenburger-Keisler Paradox, Studia Logica 86 (2007), 435-454.

[18] G. Priest, Yablo's Paradox, Analysis 57 (1997), 236-242.

[19] R.A. Sorensen, Yablo's Paradox and Kindred Infinite Liars, Mind 107 (1998), 137-155.

[20] S. Yablo, Truth and Reflection, Journal of Philosophical Logic 14 (1985), 297-349.

[21] S. Yablo, Paradox Without Self-Reference, Analysis 53 (1993), 251-252.

[22] S. Yablo, "Circularity and Paradox", in: T. Bolander, V.F. Hendricks, and S.A. Pedersen (eds.), Self-Reference CSLI Publications; 139-157, 2004.

Department of Mathematics

Behbahan Khatam Alanbia University of Technology

Behbahan, Iran

karimi@bkatu.ac.ir 